\title{
De trabajadores sociales y visitas domiciliarias en el sistema escolar chileno ${ }^{1}$
}

\author{
Alicia Razeto Pavez \\ https://orcid.org/0000-0003-2887-8424 \\ ${ }^{1}$ Pontificia Universidad Católica de Chile, Facultad de Ciencias Sociales, Escuela de Trabajo Social, Santiago, Chile (PUC)
}

\section{De trabajadores sociales y visitas domiciliarias en el sistema escolar chileno}

Resumen: Basado en la evidencia de los positivos efectos que tienen las visitas domiciliarias en el bienestar de las personas, el artículo presenta los resultados de un estudio realizado sobre la visión que tienen trabajadores sociales que se desempeñan laboralmente en escuelas públicas chilenas sobre la visita domiciliaria como estrategia de actuación profesional. Se profundiza en la definición y en la importancia que le atribuyen, en la identificación de sus límites y fortalezas y en la percepción acerca de su efectividad. La metodología del estudio fue cualitativa, con la aplicación de 3 grupos de discusión a un total de 25 trabajadores sociales.

Palabras claves: Visitas domiciliarias. Sistema escolar. Trabajadores sociales.

\section{Assistentes sociais e visitas domiciliares no sistema escolar chileno}

Resumo: Com base na evidência dos efeitos positivos que as visitas domiciliares exercem sobre o bem-estar das pessoas, o artigo apresenta os resultados de um estudo realizado acerca da visão dos assistentes sociais que atuam profissionalmente em escolas públicas chilenas sobre a visita domiciliar como estratégia de atuação profissional. Aprofunda-se na definição e na importância que lhe atribuem, na identificação de seus limites e forças e na percepção acerca de sua efetividade. A metodologia do estudo foi qualitativa, com a aplicação de três grupos de discussão em um total de 25 assistentes sociais.

Palavras-chave: Visitas domiciliares. Sistema escolar. Assistentes sociais.

\section{Social workers and home visits in the Chilean school system}

Abstract: Based on the evidence of the positive effects that home visits have on people's well-being, the article presents the results of a study about the vision of social workers who work professionally in Chilean public schools on home visits as a strategy of professional activity. It delves into the definition and importance attributed to home visits, in identifying limits and strengths, and in the perception of effectiveness. The methodology of the study was qualitative, with the application of three discussion groups involving a total of 25 social workers.

Keywords: Home visits. School system. Social workers.

Recibido en 01.08.2017. Aprobado en 08.02.2018. Revisado en 20.03.2018.

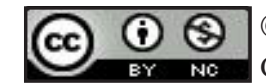

(C) El(Los) Autor(es). 2018 Acceso Abierto Esta obra está licenciada bajo los términos de la Licencia Creative Commons Atribución-NoComercial 4.0 Internacional (https://creativecommons.org/licenses/by-nc/4.0/deed.es), que permite copiar, distribuir y reproducir en cualquier medio, así como también adaptar, transformar y crear a partir de este material, desde que para fines no comerciales, y que usted fornezca el crédito debido a los autores y a la fuente, insiera un enlace para la Licencia Creative Commons e indique si fueron hechas alteraciones. 


\section{Introducción}

Estudios realizados en el campo de la salud, han determinado que las visitas domiciliarias tienen un impacto positivo en las familias. Concretamente, se han encontrado resultados positivos de las visitas domiciliarias en la salud de recién nacidos, niños y familias en riesgo social (ACADEMIAAMERICANA DE PEDIATRÍA, 1998; AVELLAR; SUPPLEE, 2013; KITZMAN et al., 1997; BAQUI et al., 2008; GOGIA; SACHDEV, 2010; CRUZ; MARTHE, 2010), embarazadas (ISSEL et al., 2011), adultos mayores (BRUGEROLLES; DOIS; MENA, 2008; MEDINA et al., 1998) y en el apoyo a los enfermos terminales (ASCENCIO-HUERTAS; RANGEL-DOMINGUEZ; ALLENDE, 2013; ASTUDILLO; MENDINUETA; GRANJA, 2008). Una de las pocas investigaciones experimentales realizadas en Chile, concluyó que las visitas domiciliarias como estrategias de intervención tienen impacto en el mejoramiento del bienestar biopsicosocial de las madres y sus hijos en un contexto de deprivación (ARACENA et al., 2011).

En el campo de la educación, son escasos los estudios aplicados sobre visita domiciliaria tanto a nivel internacional como nacional. Este déficit de investigación, no se corresponde con el alto uso de esta estrategia por parte de los trabajadores sociales que se desempeñan en escuelas ${ }^{2}$.

Este artículo tiene como propósito analizar los resultados de un estudio enfocado en la aplicación de la visita domiciliaria en las escuelas públicas, el cual tuvo como propósito analizar la visión colectiva que tienen sobre la estrategia e identificar los límites y oportunidades que les brinda en su actuación profesional. La metodología utilizada fue cualitativa, aplicando tres grupos de discusión a una muestra total de 25 trabajadores sociales de escuelas y liceos públicos de una comuna de la Región Metropolitana de Chile.

\section{Referentes conceptuales}

Históricamente, las visitas al hogar han sido utilizadas por educadores, médicos, enfermeras y trabajadores sociales como una herramienta efectiva para proveer apoyo y servicios a los niños y sus familias (COOK; SPARKS, 2008; WASIK; BRYANT, 2001). Desde un inicio, los primeros visitadores sociales, reconocieron como característica singular de la visita domiciliaria la disposición para ingresar al hogar y vecindario de una familia en señal de una relación menos formal y más relajada entre el visitador y los padres, equiparando el poder entre ambos (COOK; SPARKS, 2008).

La visita domiciliaria consiste en la entrega directa de un servicio de ayuda, información o guía a individuos en su hogar en el área de salud, apoyo social o educación (UNITED STATES OF AMERICA, 1990). De acuerdo a Aracena et al. (2011) este servicio puede estar dirigido a una persona o familia en sus distintas etapas de vida, desde antes de nacer hasta la adultez mayor. En todos los casos, la principal característica es que se lleva a cabo en el propio hogar de las personas y a través de encuentros habituales a lo largo del tiempo, presentándose como una herramienta óptima para poblaciones en condiciones de pobreza o exclusión social, por sus dificultades para acceder o desplazarse hacia los sistemas formales de atención social (ARACENAet al., 2011). En este sentido, las visitas domiciliarias contribuyen a superar barreras tales como enfermedad, discapacidad física o presencia de necesidades especiales, falta de transporte, falta de cuidado de niños, baja motivación y alienación desde el sistema educacional o médico (WASIK; BRYANT, 2001).

Wasik e Bryant (2001) definen la visita domiciliaria como un proceso mediante el cual un agente visitador otorga ayuda a una familia durante un período de tiempo extenso en su propio hogar. El agente visitador puede ser o no un profesional. Según estos autores la ayuda o el servicio se enfoca a cubrir diversas necesidades de la familia, como las de carác-

Este artículo tiene como propósito analizar los resultados de un estudio enfocado en la aplicación de la visita domiciliaria en las escuelas públicas, el cual tuvo como propósito analizar la visión colectiva que tienen sobre la estrategia e identificar los límites y oportunidades que les brinda en su actuación profesional. ter social, emocional, cognitiva, de salud y educativas.

La principal ventaja de la visita domiciliaria es que la provisión de los servicios a las familias se realiza en su ambiente natural lo que facilita la detección y comprensión de las necesidades familiares junto con la 
individualización de los servicios para responder a ellas (ALLEN; TRACY, 2004). Por otra parte, contribuye a balancear la relación asimétrica de poder entre el profesional y la familia, ayudando a construir relaciones de confianza (ALLEN; TRACY, 2004). En definitiva, las cualidades de las visitas domiciliarias consisten en ofrecer la oportunidad única de obtener información relevante acerca del ambiente familiar, sus recursos y necesidades y de entregar un servicio individualizado. Transmite, a su vez, un mensaje de respeto y apreciación por las necesidades de las familias (WASIK; BRYANT, 2001).

Los niveles en los que se realiza la entrega del servicio pueden consistir en la prevención social, la intervención o tratamiento, o la evaluación. En esta línea, Wasik e Bryant (2001) establecen diversas razones por las cuales puede iniciarse una visita domiciliaria. Pueden ser ofrecidas como servicio universal, por ejemplo, desde los centros de salud locales a padres de recién nacidos. O bien, pueden ser ofrecidas como estrategia de prevención o intervención de acuerdo a las necesidades especiales de los niños. Por ejemplo, a nivel preventivo las visitas pueden ser ofrecidas a las familias cuyos niños están en riesgo de deserción escolar. A nivel de intervención, las visitas pueden ser realizadas por profesores hacia los padres que tienen niños con retraso del desarrollo. En los dos niveles, los agentes visitadores pueden ayudar a los padres a aprender los mecanismos para mejorar el desarrollo social y cognitivo de los niños. En otras instancias, pueden ser las familias las que soliciten el servicio como ayuda en el cuidado de algún miembro con discapacidad física o enfermedad crónica. El propio sistema judicial también puede requerir que algunas familias participen en visitas domiciliarias, en casos de negligencia, casos de abuso o disputas en la custodia del niño.

Sea cual sea la razón por la que es efectuada, es fundamental que los agentes visitadores sean competentes para que la visita sea efectiva. Por tanto, debe tener ciertas características, contar con conocimientos técnicos, habilidades y con supervisión (WASIK; BRYANT, 2001). Hay cierto consenso en que sea o no profesional, el agente visitador debe estar capacitado para realizar la visita (AVELLAR; SUPPLEE, 2013; ARACENA et al., 2011; WASIK; BRYANT, 2001). Del mismo modo, las visitas domiciliarias tienen etapas y tienen que considerar aspectos prácticos como la planificación, el tiempo, los materiales, la documentación, seguridad y confidencialidad (WASIK; BRYANT, 2001).

Tal como proponen Wasik e Bryant (2001), el bienestar y desarrollo social de los niños es uno de los principales propósitos de la visita domiciliaria, por lo que el sistema escolar es un contexto idóneo para realizarlas. El potencial de aplicarlas desde el ámbito escolar, se fundamenta en que la visita domiciliaria es "una estrategia que puede facilitar la participación de los padres en la educación de sus hijos y ayudar a entender mejor el contexto extraescolar y sus influencias en el aprendizaje, cuestiones claves para las escuelas básicas". (ALLEN; TRACY, 2004).

De acuerdo a Allen e Tracy (2004) existen tres modelos de programas de visitas domiciliarias en escuelas. Según su foco, estos modelos son:

1) Enfocado en el proceso: en este modelo las visitas pueden ser realizadas para el conocimiento mutuo entre familias y profesionales de la escuela, para informar al staff acerca del contexto social y cultural de las familias, para comunicar el trabajo de la escuela a los padres y fomentar el apoyo familiar.

2) Enfocado en los recursos: en este modelo las visitas se realizan antes o durante el inicio del año escolar a las familias nuevas o de los niveles iniciales, y contemplan la referencia a agencias y servicios para responder a las necesidades familiares. Sirven para realizar las conexiones iniciales entre familias y escuelas, así como también para instalar la base para la comunicación con los padres que ocurrirá a lo largo del año.

3) Enfocado en el riesgo: aquí las visitas se realizan para incrementar la colaboración entre las familias y las escuelas en temas académicos y de comportamiento, reduciendo que ciertos grupos de niños presentes dificultades de comportamiento o académicas. Se establecen metas y monitorean actividades junto a las familias para apoyar el desempeño de los niños.

Según estos autores las visitas domiciliarias pueden mejorar la conexión entre la escuela y la familia, bajo la premisa que los padres no necesariamente tienen que ingresar a la escuela para influir e involucrarse en la educación de sus niños. Hay actividades importantes de involucramiento que pueden desarrollarse desde el hogar. Las visitas pueden ayudar a

[...] resolver las barreras de la participación de los padres en la educación de sus hijos, como lo son la falta de tiempo para ir a reuniones o para leer materiales enviados a la casa, no sentirse valorado ni bienvenido en la escuela, no comprender el sistema escolar ni su lenguaje, y la accesibilidad. (ALLEN; TRACY, 2004, p. 200).

Así es como también las visitas domiciliarias pueden proveer mejores mecanismos para responder a la 
diversidad cultural de las familias de los estudiantes. Cada cultura puede lograr el involucramiento de manera distinta o en distintos caminos, que pueden ser subvalorados o malinterpretados por la escuela. Así mismo, las visitas pueden ayudar al staff a aprender acerca de asuntos de diversidad y comunicación del trabajo de la escuela hacia las familias.

\section{Metodología}

Con un enfoque cualitativo, se aplicaron tres grupos de discusión como estrategia de recolección de información, pues se buscaba conocer la visión que tienen los trabajadores sociales sobre la visita domiciliaria en el contexto educativo.

La muestra fue intencional, compuesta por 25 trabajadores sociales de sexo femenino (18) y masculino (7) que se desempeñan laboralmente en escuelas y liceos municipales de una comuna de la Región Metropolitana, con distinta cantidad de horas y tipo de jornada.

Los grupos de discusión fueron aplicados de manera paralela, el mismo día, en el marco de una jornada de trabajo con los profesionales organizada por la Universidad y por el municipio. Cada grupo fue dirigido por un miembro parte del equipo de investigación y se aplicó en ellos la misma pauta temática. Se realizaron en tres salas de la universidad a puertas cerradas y sin interrupciones. La discusión de los grupos fue grabada - con el consentimiento de los participantes - para luego ser transcrita y analizada.

La distribución de participantes y la duración de los grupos se describen en el Cuadro 1.

\section{Cuadro 1 - Antecedentes generales de los grupos de discusión (GD)}

\begin{tabular}{c|c|c}
\hline Identificación del grupo & Cantidad de participantes & Duración \\
\hline GD1 & 8 & 47 minutos \\
GD2 & 9 & 1 hora \\
GD3 & 8 & 1 hora y 2 minutos \\
\hline
\end{tabular}

Fuente: Elaboración propia

Las categorías de análisis fueron elaboradas de manera deductiva en relación con el objetivo de la investigación. Las principales categorías fueron las siguientes:

- Definición de visita domiciliaria en educación

- Importancia de la visita domiciliaria en educación: en esta categoría surgen dos subcategorías inductivas sobre el rol del trabajador social en la escuela y su integración en equipos interdisciplinarios.

- Limitaciones de la visita domiciliaria

- Fortalezas de la visita domiciliaria

- Percepción de efectividad de la visita domiciliaria

\section{Resultados y discusión}

\section{Definición de visita domiciliaria en educación}

Todos los participantes valoraron a la visita domiciliaria como una herramienta de trabajo profesional orientada a conocer la situación del estudiante y su familia de manera completa y más profunda.

Sin embargo, se pudo observar que los trabajadores sociales restringieron esta definición a considerarla sólo como una estrategia para recabar información de la situación de la familia y el estudiante. A diferencia de lo planteado por United States of America (1990), Cook e Sparks (2008), e Wasik e Bryant (2001), quienes conceptualizan a la visita domiciliaria como un proceso que permite entregar apoyo o servicios directos en materia de salud, social o educativa, los participantes del estudio no le otorgan la cualidad de permitir un trabajo preventivo o de entrega de servicios de apoyo.

Como lo muestra la Figura 1, los participantes asociaron la visita a objetivos tales como corroborar, descubrir, contrastar, indagar, contactar y profundizar información sobre el estudiante y su realidad 
sociofamiliar con relación a las demandas de los casos que atienden referidas a situaciones de estudiantes, tales como inasistencia, maltrato, abandono o vulneración de derechos en general.

\section{Figura 1 - Objetivos de la visita domiciliaria según los trabajadores sociales participantes}

\begin{tabular}{c|c|c|c|c|c|c}
\hline \multicolumn{7}{c}{ Objetivos de la visita domiciliaria en educación } \\
\hline $\begin{array}{c}\text { Corroborar } \\
\text { información } \\
\text { familiar }\end{array}$ & $\begin{array}{c}\text { Ver qué está } \\
\text { pasando en la } \\
\text { familia }\end{array}$ & $\begin{array}{c}\text { Contrastar } \\
\text { realidades entre } \\
\text { el hogar y la } \\
\text { escuela }\end{array}$ & $\begin{array}{c}\text { Verificar en } \\
\text { terreno la } \\
\text { situacion del } \\
\text { estudiante }\end{array}$ & $\begin{array}{c}\text { Conocer más en } \\
\text { detalle o } \\
\text { profundizar la } \\
\text { situacion familiar }\end{array}$ & $\begin{array}{c}\text { Estudiar a la } \\
\text { familia y su } \\
\text { ambiente }\end{array}$ & $\begin{array}{c}\text { Entender el } \\
\text { hábitat o contexto } \\
\text { del estudiante }\end{array}$ \\
\hline
\end{tabular}

Fuente: Elaboración propia

Se le comprende como una estrategia de conocimiento, diagnostico o evaluación, complementaria con otras visiones y antecedentes recabados por otros profesionales, que permite aportar antecedentes para una futura actuación profesional posterior con dichas familias.

Es una herramienta para poder profundizar en ciertos casos donde no podemos visualizar según los antecedentes, ya sea que levantó el profesor, que levantó la psicóloga y que necesitamos profundizar y tener más detalles respecto a la dinámica familiar, las condiciones donde vive el niño, etc., principalmente para profundizar y la cual pueda ayudarnos para la intervención o las acciones que vamos a seguir con esas familias. (GD3).

Una de las razones que con mayor frecuencia mencionaron como causa de la aplicación de la visita fue la inasistencia del estudiante a la escuela, lo que coincide con lo planteado por Wasik e Bryant (2001) como una de las causas que pueden originar la utilización de la visita domiciliaria: "Yo la hago, en lo personal, cuando hay muchas ausencias y ninguna de las vías funcionan, como la comunicación o el llamado telefónico, o problemas socioeconómicos donde uno tiene que ir para luego hacer el informe social y derivar". (GD2).

A su vez, los participantes mencionaron su ventaja ante la presencia de ciertas barreras para acceder a las familias, como los problemas económicos, o la inefectividad de otros mecanismos, como el llamado telefónico. Esto es convergente con lo planteado por Allen e Tracy (2004), Wasik e Bryant (2001) e Aracena et al. (2011).

\section{Importancia de la visita domiciliaria en educación}

Los participantes le asignaron relevancia a la visita domiciliaria. La mayoría consideró importante su utilización para potenciar el vínculo con las familias de los estudiantes en su espacio más íntimo. La Figura 2 describe las valoraciones otorgadas a la visita por parte de los profesionales:

Figura 2 - Valoraciones a la visita domiciliaria por parte de los participantes

\begin{tabular}{l} 
Relevancia de la visita domiciliaria \\
\hline - Herramienta potente para el acercamiento con las familias \\
- Les valida ante los padres porque lo agradecen \\
- Fundamental para corroborar hipótesis de trabajo \\
- Genera otro tipo de vínculo con las familias \\
- Permite que las familias se sientan en su propio espacio \\
\hline
\end{tabular}

Fuente: Elaboración propia 
En sintonía con lo planteado por Allen e Tracy (2004) los participantes aprecian la capacidad que tiene para estrechar la relación con las familias de los estudiantes, en un contexto menos masivo y de mayor confianza como lo representa el hogar del estudiante: "En los colegios grandes los papás sienten que los niños pasan a ser uno más de los miles que hay, entonces que uno vaya a la casa, hay papás que lo agradecen mucho”. (GD1).

Este aspecto permitiría tener acceso a información privilegiada, que no se logra tener en el contexto de la escuela. "Uno genera otro tipo de vínculo con la familia, un acercamiento diferente y puede ver otras cosas que no se ven en el colegio." (GD3).

Para Strom-Gottfried (2009) esta exposición a la información es una ventaja de los servicios basados en el hogar, pero también representa desafíos de naturaleza ética, puesto que dicha información el profesional la utilizará para decidir activar ciertos procesos de ayuda. Por otro lado, las impresiones de las condiciones de vida y experiencias poderosas experimentadas por el agente visitador pueden afectar el establecimiento de límites apropiados, disminuyendo la capacidad para enfocarse en el propósito profesional y haciendo más probable la aparición del síndrome de bournout. Este tipo de aspectos éticos no fueron discutidos por los trabajadores sociales participantes del estudio.

En la discusión sobre la importancia de la visita domiciliaria, surgió como asunto de interés para los participantes problematizar el rol que cumplen en las escuelas. En esto hubo consenso en catalogar su rol como difuso, en tanto los desempeños esperados son poco conocidos y las funciones que asumen dependen de la cultura institucional del establecimiento educacional. Esta falta de claridad no concuerda con la literatura, en la que se destaca el rol de los trabajadores sociales en el apoyo a los resultados educativos positivos de los estudiantes a través de la influencia y coordinación de los esfuerzos de la escuela, familia y comunidad (FRANKLIN; GERLACH; CHANMUGAM, 2008; AMERICAN COUNCIL FOR SCHOOL SOCIAL WORK, 2015). En esta línea, Allen e Tracy (2004) describen a los trabajadores sociales como un puente clave entre la escuela y los hogares, promoviendo el involucramiento familiar en la educación de los niños y ayudando al staff de la escuela a comprender y relacionarse con la diversidad de formas familiares, estilos de vida y contextos.

A propósito de la falta de claridad en el rol que deben asumir los trabajadores sociales en las escuelas, en uno de los grupos se propone que las instituciones académicas asuman una función activa que ayude a determinar el quehacer de los profesionales en el área educacional: "Uno apuesta también a que la academia haga un aporte desde la mirada, justamente más técnica, de la cual podría ser el rol del trabajador social en las escuelas, que es un rol medio difuso, que cada uno lo va haciendo desde su nicho". (GD2).

Los participantes destacan la relevancia que tiene en su quehacer profesional la relación con otros profesionales presentes en las escuelas, enfatizando en la interdependencia y a la vez en la necesidad de mejorar la coordinación entre éstos. En los grupos se destaca al director o directora como una pieza fundamental porque contribuye a establecer límites con los apoderados; a los inspectores como agentes que suelen acudir al trabajador social para pedir apoyo; a los profesores como importantes pero resistentes al trabajo con ellos por su exigida situación laboral; a los paradocentes como relevantes aunque suelan no ser considerados. Especial cercanía de trabajo se produce con los psicólogos, los orientadores y los jefes de Unidad Técnico Pedagógica, siendo los aliados del quehacer profesional de los trabajadores sociales.

\section{Limitaciones y dificultades de la visita domiciliaria en educación}

Los participantes identifican un conjunto de limitaciones de carácter práctico u operativo en la aplicación de la visita domiciliaria en la educación, los cuales se listan a continuación.

1. Sensación de inseguridad del profesional en algunos de los barrios donde se realiza la visita domiciliaria, en relación a la vulnerabilidad social de los hogares donde provienen los estudiantes: "[...] tiene que ver también con que nuestros alumnos son vulnerables, y los lugares donde residen no sé si son peligrosos, pero generan cierta inseguridad”. (GD1).

Esta limitación tiene cierto asidero en la literatura, puesto que según Puls (2006) todo profesional que visita a una persona o familia en su hogar, está expuesto a un riesgo o inseguridad del cual debe ser consciente y desarrollar conductas para encontrar soluciones. Sin embargo, los participantes del estudio asocian este peligro a la situación socioeconómica de las familias, creencia que puede ser errónea porque "que una persona viva en un área central, en una casa grande y hermosa, eso no significa que la persona no tenga algún desequilibrio mental, problema con drogas o tendencias violentas". (PULS, 2006, p. 15).

2. Dificultad en el desplazamiento del trabajador social hacia los hogares de los estudiantes, por la 
dispersión geográfica y por la falta de tiempo profesional y de recursos económicos para costear traslado, como lo demuestra la siguiente cita: “[...] una limitación súper práctica es el tema de las lucas para viajar, quién me paga la bencina, eso”. (GD2).

3. Sensación de invasión de privacidad por parte de las familias y de control hacia ellas, que de no ser cautelado, podría generar distancia en los padres:

[...] la privacidad es rota por una serie de agentes de control social, entre ellos nosotros y, por lo tanto, tenemos que ser cuidadosos, no digo que no lo hagamos, pero en aras de no saturar las entradas a las historias vitales de esos grupos que entra uno, dos, tres, cuatro, cinco actores [...] (GD2).

4. Observación de sólo un momento parcial de la situación e historia de la familia, comprendido por el período de realización de la visita, que suele ser menor a los 60 minutos: "[...] uno ve unos minutos de la realidad no más, que no es lo mismo, que monitorear al niño en clases, durante varias semanas". (GD1). Esto se suma a la falta de continuidad en la realización de visitas domiciliarias para un mismo estudiante.

\section{Oportunidades y fortalezas de la visita domiciliaria en educación}

En concordancia con la importancia asignada a la visita, los participantes del estudio identifican oportunidades y fortalezas, relacionadas al vínculo entre el profesional, estudiante y adulto responsable. Entre las principales fortalezas de la visita están:

1. Posibilita espacios de conversación más profundos y genera vínculos afectivos y de confianza entre profesional-padres, padres-estudiante, adulto responsable-estudiante:

Yo creo que la visita mejora el vínculo con la familia, yo creo que eso es lo más relevante de la visita domiciliaria [...] las personas se sienten agradecidas que uno esté en su domicilio, que haya llegado hasta su casa que cuesta un montón llegar y que uno esté ahí viendo con ellos su contexto, cómo viven, cuáles son sus condiciones, etc. (GD1).

2. Permite desahogar las opiniones y sentimientos de los padres que carecen de redes de apoyo para resolver sus problemas: "A veces las visitas se transforman en un espacio de desahogo para los papás porque no tienen red de apoyo familiar a quien contarle sus problemas". (GD1).

3. Genera instancias de acercamientos con las familias previos a reuniones o entrevistas con otros actores de los establecimientos educacionales, como lo ejemplifica la siguiente cita:

Cuando hay casos de estudiantes disruptivos los inspectores generales acuden a mi para que vaya a la casa a generar otro vínculo, para que no sea tan compleja como la entrevista con los inspectores en el liceo [...] Se logra un vínculo previo, obviamente la situación es compleja, pero ya es distinta la relación en la casa y tratando de mediar antes de que apoderado llegue a la entrevista con el inspector general donde le va a mostrar las cinco hojas de anotaciones que tiene el estudiante y lo que eso conlleva. (GD1).

4. Ayuda a favorecer los resultados educativos de los estudiantes, a través del incentivo de la asistencia a clases: "La vinculación de la madre con la familia, cuando no está asistiendo a clases y tu hiciste la visita, ya como última circunstancia y después empieza a venir el niño y tú te das cuenta 'chuta esto igual funcionó y la familia se acerca más a uno". (GD2).

\section{Percepción de efectividad de la visita domiciliaria}

Los participantes fueron consultados sobre si perciben cambios en el estudiante o sus familias una vez realizada la visita domiciliaria, ante lo cual respondieron detectar principalmente cambios positivos, siendo el más importante el vínculo que se genera entre las familias y el trabajador social. Los efectos positivos de la visita domiciliaria percibidos por los participantes fueron los siguientes:

1. Desarrollo de un vínculo entre apoderados y el trabajador social. 
2. Más presencia, compromiso y atención de las familias hacia los estudiantes.

3. Desarrollo de una relación colaborativa entre familias y escuela.

4. Valoración por parte de las familias del interés demostrado por la escuela por sus estudiantes en un ámbito distinto al escolar.

Estos efectos no han sido medidos sino que corresponden a relatos basados en sus experiencias de actuaciones profesionales, sin estudios ni evaluaciones de su impacto. Ello dista de la realidad internacional, en la cual los aportes y potencialidades de los trabajadores sociales educacionales quedan de manifiesto a partir de diversas mediciones. Por ejemplo, un estudio de meta-análisis de 21 publicaciones de estudios norteamericanos sobre la efectividad de las prácticas del trabajo social escolar entre 1980 y 2007 (FRANKLIN; KIM; TRIPODI, 2009) arrojó resultados que "iluminan el impacto positivo que los trabajadores sociales de escuelas pueden tener en los resultados académicos, conductuales, mentales y emocionales de los estudiantes". (FRANKLIN; KIM; TRIPODI, 2009, p. 674, traducido).

La visita domiciliaria permitiría producir un cambio en las actuaciones de los padres, aumentando su compromiso respecto a la situación del estudiante. Por ejemplo, los padres incrementarían su presencia a través de los llamados telefónicos a la escuela para percatarse de si el estudiante ha asistido a la escuela. A su vez, se aprecia que la visita domiciliaria impacta positivamente en la disposición de los padres hacia la escuela, pues se percatan de la preocupación que existe hacia ellos.

No sé si en la sala de clases haya algún cambio, pero a mí en lo que he visto en la vinculación con los papás o del adulto significativo que esté a cargo del niño hay un cambio, hay mayor compromiso, después se trabaja de otra forma, están más abiertos. En la vinculación se genera un cambio. (GD3).

A pesar de lo positivo que puede llegar a resultar la aplicación de la visita, los participantes también manifestaron que podría tener efectos indeseables. Pero esto solo se reconoció en uno de los grupos de discusión, donde algunos relataron experiencias personales vividas y/o supuestos de lo que consideraban la visita domiciliaria provocaría. Entre los efectos negativos se mencionan la situación de amenaza percibida por algunas familias ante la presencia de un profesional en sus hogares, que podría originar un cambio de colegio.

En la familia particularmente yo lo noto más y siempre hay uno, puede no ser favorable a veces que me ha ocurrido, por ejemplo, con los días han cambiado al niño de colegio porque se sienten amenazados $y$ tienen además una actitud, a veces muy pasiva que no te hacen entender que ellos van a tomar esa medida. (GD2).

También se menciona como efecto indeseado el sentimiento de vergüenza de las familias por la presencia de un profesional de la escuela en sus hogares y la observación de su espacio íntimo, lo que podría desencadenar un distanciamiento de las familias con la escuela.

\section{Consideraciones finales}

El artículo muestra los resultados de un estudio pionero en Chile que tuvo como objeto de investigación a la visita domiciliaria realizada en contexto escolar por parte de trabajadores sociales. El estudio tuvo como propósito analizar la visión que tenían un grupo de trabajadores sociales del sistema escolar sobre la visita domiciliaria utilizada en su contexto profesional, descubriendo sus definiciones, límites y oportunidades.

En la definición de la visita domiciliaria, los participantes la visualizaron como una herramienta profesional dirigida a estudiar el contexto familiar del estudiante, sin considerarla como una estrategia mediante la cual es posible entregar un servicio de apoyo directo en distintas materias. Esto sugiere una subutilización de la visita domiciliaria en el contexto educacional, al no ser utilizada como un recurso para el apoyo directo a las familias y al estudiante, con miras a incrementar la participación de los padres en la educación de sus niños y favorecer el resultado educacional.

En la importancia atribuida a la estrategia, los trabajadores sociales destacan su bondad en términos de la posibilidad de conseguir información más profunda del grupo familiar y del establecimiento de un mejor vínculo con las familias en un espacio de mayor confianza. Por otra parte, la discusión de los grupos enfatizó en la poca claridad del rol profesional que asumen en las escuelas así como también a la necesaria conexión que deben establecer con otros profesionales de la escuela. De algún modo, esto sugiere la necesidad de brindar más y mejores espacios para la formación profesional de los trabajadores sociales en el campo de la educación. 
Si bien éste se presenta como un ámbito creciente de inserción profesional, a nivel nacional no existen programas de formación profesional que aborden los conocimientos y habilidades que tiene que desarrollar un profesional en el campo de la educación.

Respecto a las limitaciones de la visita domiciliaria, los participantes destacaron la inseguridad que significaba aplicarlas en barrios vulnerables, así como también la falta de recursos económicos y de tiempo para el desplazamiento a los hogares de los niños. También se mencionaron la discontinuidad de la visita para los casos seleccionados y el escaso tiempo invertido en ellos. Con relación a sus principales fortalezas, se destacó su capacidad de generar vínculos estrechos de mayor confianza con las familias así como también su positiva incidencia en casos de inasistencia escolar.

Con relación a la percepción de efectividad, ninguno de los participantes señaló haber realizado estudios concretos, pero sí mencionaron percibir efectos positivos en la mayor presencia y compromiso de los padres en la educación de sus hijos, incidiendo en el desarrollo de una relación colaborativa entre la escuela y las familias. Sin embargo, los trabajadores sociales reconocen no tener certezas de si estos cambios positivos llegan a manifestarse dentro de la sala de clases. Potencialmente, la visita podría conllevar ciertos efectos no deseados como el distanciamiento de la familia, por sentirse amenazados o avergonzados de su propio contexto. Esto realza la relevancia que tiene la capacitación del profesional en la aplicación de esta estrategia.

La principal fortaleza del estudio es poner foco a una herramienta profesional que a pesar de ser tan cercana históricamente a la profesión del Trabajo Social, ha sido muy poco estudiada en términos aplicados. Al mismo tiempo, contribuye a sensibilizar a los trabajadores sociales sobre las potencialidades que tiene la visita domiciliaria en el campo educacional en términos del incremento de la participación de las familias en la educación de los niños. Para finalizar, es preciso mencionar las limitaciones del estudio, las cuales consisten en el reducido tamaño de la muestra y su carácter intencionado, que impiden generalizar los resultados a otras poblaciones.

\section{Referencias}

ACADEMIA AMERICANA DE PEDIATRÍA. The Role of Home Visitation Programs in Improving Health Outcomes for Children and Families. Pediatrics, Columbus, v.3, n. 101, p. 486-490, 1998.

ALLEN. S.; TRACY, E. Revitalizing the Role of Home Visiting by School Social Workers. Children \& Schools, Oxford, v. 26, n. 4, p. 197-207, 2004.

AMERICAN COUNCIL FOR SCHOOL SOCIAL WORK. About School Social Work. Disponible en: < http://acssw.org/ aboutschool.html>. Acceso en: 5 de marzo de 2016.

ARACENA, M.; et al. Evaluación de la efectividad de programas de visitas domiciliarias para madres adolescentes y sus hijos/as. Revista Médica de Chile, Santiago, vol. 139, n. 139, p. 60-65, 2011.

ASCENCIO-HUERTAS, L.; RANGEL-DOMÍNGUEZ, N.; ALLENDE, S. Proceso de atención psicológica en el programa de atención y cuidados en oncología del Instituto Nacional de Cancerología. Psicooncología, Madrid, v. 10, n. 2-3, p. 393-406, 2013.

ASTUDILLO, W.; MENDINUETA, C.; GRANJA, P. Cómo apoyar al cuidador de un enfermo en el final de la vida. Psicooncología, Madrid, v. 5, n. 2-3, p. 359-381, 2008.

AVELLAR, S.; SUPPLEE, L. Effectiveness of Home Visiting in Improving Child Health and Reducing Child. Pediatrics, Columbus, v. 132, n. 2, 2013.

BAQUI, A.; et al. Impact of an integrated nutrition and health programme on neonatal mortality in rural northern India. Bulletin of the World Health Organization, Geneva, v. 86, n. 10, p. 796-805, 2008.

BRUGEROLLES, M.; DOIS, A.; MENA, C. Beneficio de la visita domiciliaria para los adultos mayores. Horizonte de Enfermería, Santiago, v. 19, n. 2, p. 55-64, 2008.

COOK, R.; SPARKS, S. The art and practice of home visiting. Maryland: Brookes, 2008.

CRUZ, M.; MARTHE, M. A. Visita Domiciliária na Estratégia de Saúde da Família: conhecendo as percepções das famílias. Saúde e Socidade, São Paulo, v. 19, n. 3, p. 605-613, 2010.

FRANKLIN, C.; GERLACH, B.; CHANMUGAM, A. School social work. In: SOWERS, K.; DULMUS, C. Comprehensive Handbook of Social Work and Social Welfare. New Jersey: John Wiley \& Sons, 2008. p. 205-226.

.; KIM, J.; TRIPODI, S. A Meta-Analysis of Published School Social Work Practice Studies 1980-2007. Research on Social Work Practice, Tallahassee, v. 19, n. 6, p. 667-677, 2009.

GOGIA, S.; SACHDEV, H. S. Home visits by community health workers to prevent neonatal deaths in developing countries: a systematic review. Bulletin of the World Health Organization, Geneva, v. 88, n. 9, p. 658-666B, 2010.

UNITED STATES OF AMERICA. Government Accountability Office. Home Visiting: a promising early intervention strategy for atrisk families. Washington, D.C., 1990. Disponible en: < https://www.gao.gov/products/HRD-90-83>. Acceso en: 5 de marzo de 2018. ISSEL, L. et al. A Review of Prenatal Home-Visiting Effectiveness for Improving Birth Outcomes. JOGNN, vol. 2, n. 40, p. 157-165, 2011. 
KITZMAN, H. et al. Effect of prenatal and infancy home visitation by nurses on pregnancy outcomes, childhood injuries, and repeated childbearing. A randomized controlled trial. JAMA, Chicago, v. 278, n. 8, p. 644-652, 27 Aug. 1997.

MEDINA, M. et al. Evaluación del impacto en cuidadores de usuarios del Servicio de Ayuda a Domicilio. Anales de Psicología, Murcia, v. 14, n. 1, p. 105-126, 1998.

PULS, C. Safe Home Visits: powerful information you can use immediately to make your work inside the client's home much safer. Bloomington: Trafford Publishing, 2006.

STROM-GOTTFRIED, K. Two ethical issues and guidelines. In: Allen, S; Tracy, E. Delivering Home-Based Services: A Social Work Perspective. Columbia: Columbia University, 2009. p. 14-33.

WASIK, B.; BRYANT. D. Home visiting. Thousand Oaks: Sage, 2001.

\section{Notas}

1 Este artículo es un producto de la investigación FONDECYT de Iniciación año $2014 \mathrm{n}^{\circ} 11140679$ titulada La visita domiciliaria como estrategia de intervención social para aumentar la participación de los padres en la educación de sus hijos en escuelas básicas municipales.

2 Durante los últimos cinco años, a partir de la incorporación de la Ley de Subvención Escolar Preferencial - que adiciona nuevos recursos a las escuelas - se ha acrecentado la presencia de los trabajadores sociales contratados en los establecimientos educativos chilenos.

\section{Alicia Razeto Pavez}

arazeto@uc.cl

Doctorado en Ciencias de la Educación por la Pontificia Universidad Católica de Chile (UC)

Académica de la Escuela de Trabajo Social de la Facultad de Ciencias Sociales de la Pontificia Universidad Católica de Chile (UC)

\section{$\mathbf{U C}$}

Escuela de Trabajo Social

Avenida Vicuña Mackenna, 4860 - Macul

Santiago - Provincia de Santiago - Chile

Código Postal: 7820436

\section{Agencia financiadora}

Fondo Nacional de Desarrollo Científico y Tecnológico (FONDECYT), dependiente de la "Comisión Nacional de Investigación Científica y Tecnológica (CONICYT)” del Gobierno de Chile. Proyecto n. 11140679.

Período de ejecución: de enero de 2015 hasta febrero de 2018.

\section{Contribuciones de los autores}

No se aplica.
Aprobación por Comisión de Ética y consentimiento para participación

No se aplica.

Consentimiento para publicación

No se aplica.

\section{Conflicto de intereses}

No se declaran conflictos de intereses. 